\title{
Dexamethasone Treatment for COVID-19-Related Lung Injury in an Adult with WHIM Syndrome
}

\author{
Daigo Akahane $^{1}$ (D) Shunsuke Otsuki ${ }^{1} \cdot$ Daisuke Hasegwa $^{2} \cdot$ Hidehiro Watanabe $^{3} \cdot$ Akihiko Gotoh $^{1}$
}

Received: 22 May 2021 / Accepted: 22 November 2021 / Published online: 1 December 2021

(c) The Author(s), under exclusive licence to Springer Science+Business Media, LLC, part of Springer Nature 2021

To the editor,

Coronavirus disease 2019 (COVID-19), a new type of coronavirus infection caused by severe acute respiratory syndrome coronavirus 2 (SARS-CoV-2), was first reported in China in December 2019. Elderly people and patients with underlying diseases often become severely ill with this disease. Indeed, as of 2021, COVID-19 has spread worldwide, becoming a major social concern. For patients with blood disorders and immunodeficiency, the development of severe symptoms due to COVID-19 is of concern, although sufficient data regarding this aspect are not available.

We report the case of a patient with COVID-19 infection with WHIM syndrome diagnosed during adulthood. The patient consented to the use of his medical data. To the best of our knowledge, this is the first report of COVID-19 in a patient with preexisting WHIM syndrome. WHIM syndrome is characterized by warts, hypogammaglobinemia, infections, and myelokathexis (with peripheral blood neutropenia, but hyperplasia in the bone marrow), and is classified as a hereditary neutropenia. It is inherited through autosomal dominant inheritance and is caused by the mutation of CXCR4. Gain-of-function CXCR4 mutation, which inhibits not only the differentiation of the myeloid lineage, but also the maturation of lymphocytes, results in immunodeficiencyinduced infection [1]. Some patients with WHIM syndrome develop severe immunodeficiency from birth, whereas asymptomatic patients diagnosed in adulthood have also been reported [2].

Daigo Akahane

daigoakahane@msn.com

1 Department of Hematology, Tokyo Medical University, 6-7-1 Nishishinjuku, Shinjuku-ku, Tokyo 160-0023, Japan

2 Department of Pediatrics, St. Luke's International Hospital, Tokyo, Japan

3 Department of Infection Prevention and Control, Tokyo Medical University Hospital, Tokyo, Japan
The patient was a 37-year-old man who was diagnosed with unexplained congenital neutropenia at the age of 10 years. He frequently developed infections associated with neutropenia that required hospitalization since childhood, although he was not definitively diagnosed. His parents and siblings did not have neutropenia. He had no history of regular hospital visits since becoming an adult, but was hospitalized for hemolytic streptococcal sepsis at the age of 32. Reassessment was performed at the time of hospitalization after developing an intraarticular abscess at the age of 37 years. Bone marrow examination revealed hyperplasia of the bone marrow and dysplasia of the myelocytic series. In neutrophils, filaments between the lobulated nuclei were markedly long, thin, and irregular, and abnormal lobulation was observed (Supplemental Fig. 1). In addition, one leaf was small and had pyknosis. There were also cytoplasmic vacuoles. There was no increase in blasts and he had a normal karyotype. Bone marrow findings included dysplasia different from MDS. WHIM syndrome was suspected based on the morphology and CXCR4 gene analysis was performed by hybrid capture-based next-generation sequencing with mononuclear cells from his peripheral blood. A heterozygous mutation in CXCR4, c.1000C > T p.Arg334X, was identified and WHIM syndrome was diagnosed. The neutrophil count was approximately $100-500 / \mu \mathrm{L}$, accompanied by lymophocytepenia $(600 / \mu \mathrm{L})$ and hypogammaglobinemia (IgG $565 \mathrm{mg} / \mathrm{dL}$ ). Although this patient had a history of infection associated with neutropenia, there was no episode of intractable viral infection and the patient was followed up with supportive therapy alone. At the time of infectious complications, his leukocyte count increased to $7000-8000 / \mu \mathrm{L}$. For this reason, reported treatments for WHIM syndrome, such as regular administration of G-CSF, IVIG, and prophylactic antibiotics, were not performed. In addition, no warts were found. The patient was to be a de novo case of WHIM diagnosed in adulthood. The reasons for the delayed diagnosis were relatively few infections, no HPV disease, tendency for neutropenia to correct with severe bacterial infection, and delayed examination of bone marrow. 
One year after this diagnosis, he visited our hospital with upper respiratory tract symptoms and fever after close contact with a COVID-19 patient. At the time of visit, the reverse transcriptase-polymerase chain reaction (RT-PCR) test for SARS-CoV-2 in his nasopharyngeal swab specimens was positive; thus, he was hospitalized. He did not get a COVID-19 vaccination prior to hospitalization. There was no risk of aggravation of COVID-19 other than WHIM syndrome such as obesity and diabetes. On admission, lung CT revealed slight shadows (Fig. 1A) and he had a continuous high fever, but oxygenation was maintained. Although there was no oxygen requirement on day 6 of hospitalization, chest X-ray and chest $\mathrm{CT}$ indicated the exacerbation of pneumonia (Fig. 1B). On the day 8, an increase in respiratory rate was observed, he developed shortness of breath, and oxygen saturation with room air decreased to 93 to $94 \%$, suggesting further exacerbation of pneumonia (Fig. 1C, E). Therefore, the administration of dexamethasone at $6 \mathrm{mg} /$ day was started (Fig. 1E). After the initiation of dexamethasone administration, his fever reduced and oxygenation improved markedly, and he was discharged on day 13 (Fig. 1E). Dexamethasone was orally administered at $6 \mathrm{mg}$ for 10 days. His respiratory status improved and no medications for COVID-19, such as remdesivir, were used during the course. No anticoagulant was used as there was no increase in D-dimer. He was discharged on day 13. CT images taken after discharge demonstrated marked improvement in the lung shadows (Fig. 1D). SARS-CoV-2 PCR was negative and SARS-CoV-2 nucleocapsid antibody IgG was positive on day 30 after discharge.

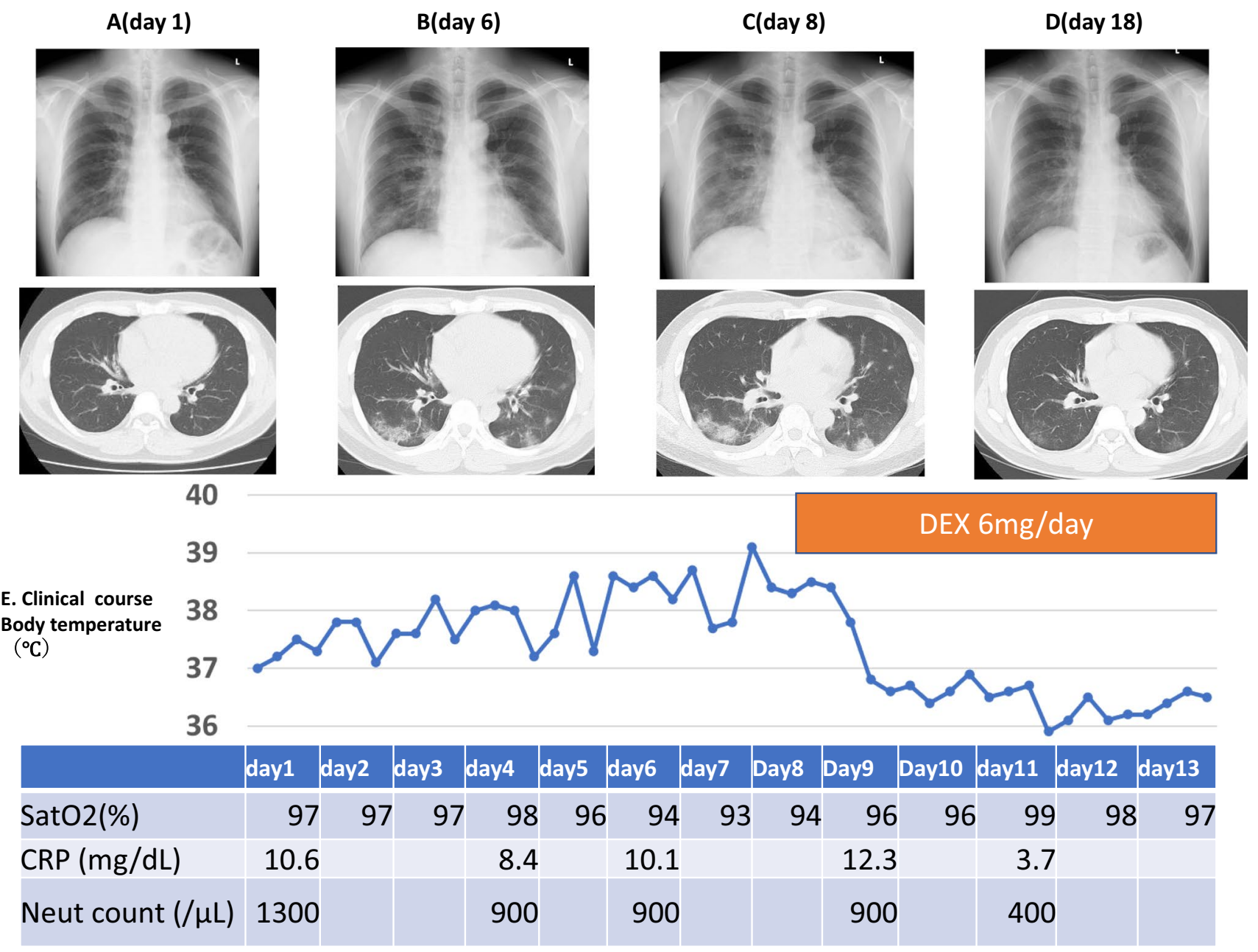

Fig. 1 The shadows were typical pulmonary manifestations of COVID-19 pneumonia, observed as ground glass opacities accompanying consolidations that primarily involved the periphery of the bilateral lower lobes. The administration of dexamethasone improved the shadows. The neutrophil count was slightly higher than normal at the onset of COVID-19. A Day 1 of admission, B day 6, $\mathbf{C}$ day 8, D day 18. $\mathbf{E}$ The clinical course of the patient infected with COVID-19. He had a persistent high fever, poor oxygenation, and high CRP. The administration of dexamethasone improved all symptoms 
Although clinical trials have been conducted with multiple drugs for the treatment of COVID-19, no fundamental therapeutic drug has been established to date. Several reports suggested the usefulness of dexamethasone in preventing respiratory failure [3]. However, patients in whom dexamethasone was effective are limited to those with moderate or higher disease requiring oxygen administration, and the administration of dexamethasone may indicate a poorer prognosis. In the early stage of disease onset, when the viral load is high, immunosuppression by the administration of dexamethasone may be a disadvantage in treating infectious diseases. However, the anti-inflammatory effects of dexamethasone may be effective against immunological lung damage that develops in the later stage of the disease. Dexamethasone suppresses the mobilization of neutrophils to the site of inflammation. Recently, dexamethasoneinduced leucine zipper gene was reported to be essential for the inhibition of neutrophil migration into inflamed tissues during dexamethasone treatment [4]. As CXCR4 is essential for neutrophil chemotaxis, in WHIM syndrome, in which its function is upregulated, dexamethasone may effectively suppress lung injury due to neutrophil migration into the lungs. It has been reported that the viral load of SARSCoV-2 peaks and slightly decreases within 1 week of disease onset in patients with normal immunity [5]. Therefore, the anti-inflammatory effects of dexamethasone can be expected without causing exacerbation. This is the first report of the onset of COVID-19 in WHIM syndrome. WHIM syndrome is known to involve abnormal immune responses to viruses due to abnormal lymphocyte differentiation, and the immunodeficiency may lead to neoplastic diseases caused by HPV and EBV. In this patient, despite their younger age, the lung shadows were more severe, with deteriorating oxygenation. As the patient was immunocompromised, exacerbation of infection due to the administration of dexamethasone was of concern, although in this case, dexamethasone inhibited the progression to respiratory failure. Basic studies revealed that the pathway mediated by $C X C R 4$ is an important factor for lung injury and pulmonary fibrosis [6]. When dexamethasone was administered from day 8 of disease onset, improvements in oxygenation, radiological findings, and fever were noted. It may also have reduced the potential risk of CXCR4-derived lung injury in WHIM syndrome. Dexamethasone may be involved in T-cell activation in CXCR4 signaling; however, whether dexamethasone administration for WHIM syndrome resulting from CXCR4 abnormalities is a negative factor for viral infection is unclear [7]. In the future, the administration of a vaccine against COVID-19 is expected to prevent disease, but in WHIM syndrome, the effects of the vaccine cannot be fully expected due to the resulting decrease in immune response. The effectiveness of CXCR4 antagonists Plerixafor and Mavorixafor has also been reported in WHIM syndrome, and improvement in the immune response against bacterial and viral infections is expected from this approach. The use of these drugs during the COVID-19 pandemic may be effective for disease control.

Abbreviations/Acronyms SARS-CoV-2: Severe acute respiratory syndrome coronavirus 2; COVID-19: Coronavirus disease 2019; CXCR4: C-X-C chemokine receptor type 4; WHIM: Warts, hypogammaglobulinemia, infections, myelokathexi

Supplementary Information The online version contains supplementary material available at https://doi.org/10.1007/s10875-021-01185-2.

Acknowledgements We would like to acknowledge Natsuko Inagaki at the Department of Clinical Genetics Center, Tokyo Medical University, as a genetics expert, for performing the genetic analysis.

Author Contribution DA and AG designed the study and wrote the manuscript. All authors contributed to the patient's care, data collection, and drafting and revision of the manuscript.

\section{Declarations}

Conflict of Interest AG received scholarships from Eisai, Ono Pharmaceutical, Taiho Pharmaceutical, Takeda Pharmaceutical, Nippon Shinyaku, Chugai Pharmaceutical, MSD, Otsuka Pharmaceutical, Sumitomo Dainippon Pharma, Nippon Shinyaku, Bayer, Daiichi-Sankyo, and Nihon Pharmaceutical, and received remuneration for time and effort spent by the researcher for meeting attendance from Novartis Pharma, Alexion Pharma, Eisai, Ono Pharmaceutical, Taiho Pharmaceutical, Takeda Pharmaceutical, Nippon Shinyaku, Chugai Pharmaceutical, Otsuka Pharmaceutical, Sumitomo Dainippon Pharma, Daiichi-Sankyo, Nihon Pharmaceutical, Kyowa Kirin, Janssen Pharmaceutical, Pfizer, and Sanofi. All other authors have no conflicts of interest to disclose.

\section{References}

1. Hernandez PA, Gorlin RJ, Lukens JN, Taniuchi S, Bohinjec $\mathrm{J}$, Francois F, et al. Mutations in the chemokine receptor gene CXCR4 are associated with WHIM syndrome, a combined immunodeficiency disease. Nat Genet. 2003;34:70-4. https://doi.org/10. 1038/ng 1149 .

2. Heusinkveld LE, Majumdar S, Gao J-L, McDermott DH, Murphy PM. WHIM syndrome: from pathogenesis towards personalized medicine and cure. J Clin Immunol. 2019;39(6):532-56. https:// doi.org/10.1007/s 10875-019-00665-w.

3. Horby P, Lim WS, Emberson JR, Mafham M, Bell JL, Linsell L, et al. Dexamethasone in hospitalized patients with COVID-19. N Engl J Med. 2021;384:693-704. https://doi.org/10.1056/NEJMo a2021436.

4. Ricci E, Ronchetti S, Pericolini E, Gabrielli E, Cari L, Gentili M, et al. Role of the glucocorticoid-induced leucine zipper gene in dexamethasone-induced inhibition of mouse neutrophil migration via control of annexin A1 expression. FASEB J. 2017;31:305465. https://doi.org/10.1096/fi.201601315R.

5. Cevik M, Tate M, Lloyd O, Maraolo AE, Schafers J, Ho A. SARSCoV-2, SARS-CoV, and MERS-CoV viral load dynamics, duration of viral shedding, and infectiousness: a systematic review and meta-analysis. Lancet Microbe. 2021;2(1):e13-22. https://doi.org/ $10.1016 / \mathrm{S} 2666-5247(20) 30172-5$. 
6. Xu J, Mora A, Shim H, Stecenko A, Brigham KL, Rojas M, et al. Role of the SDF-1/CXCR4 axis in the pathogenesis of lung injury and fibrosis. Am J Respir Cell Mol Biol. 2007;37:291-9. https:// doi.org/10.1165/rcmb.2006-0187OC.

7. Ghosh MC, Baatar D, Collins G, Carter A, Indig F, Biragyn A, et al. Dexamethasone augments CXCR4-mediated signaling in resting human $\mathrm{T}$ cells via the activation of the Src kinase Lck. Blood. 2009;113:575-84. https://doi.org/10.1182/ blood-2008-04-151803.

Publisher's Note Springer Nature remains neutral with regard to jurisdictional claims in published maps and institutional affiliations. 